\title{
Occurrence and genetic structure of Epichloë festucae endophytes in wild red fescue populations and their seed progenies
}

\author{
KARI SAIKKONEN, PIIPPA WÄLI and MARJO HELANDER \\ Agrifood Research Finland, Plant Production Research, Karilantie 2 A, FI-50600 Mikkeli, Finland
}

In the systemic grass endophyte associations only one fungal genotype is transmitted vertically to seed progeny, promoting stable interactions between the fungal genotype and the host lineage. Thus in theory, vertical transmission should align the interests of partners so that the fungus-host association should evolve toward mutualism, and frequencies of infected grasses should increase over time in plant populations, if endophytes increase fitness of their host. Evidence from agricultural grasses generally also supports this prediction. Our studies (as well as few others) with seminatural and natural, native grass endophyte systems reveal, however, that endophyte-plant interactions are much more complex than described in past literature and represent a diverse continuum from antagonistic to mutualistic interactions.

We examined (1) the infection frequency and genotypic diversity of systemic endophyte (Epichloë festucae) in wild red fescue (Festuca rubra) populations in northernmost Finland, and then (2) in a series of laboratory and common garden experiments, we tested whether endophyte-plant associations are affected by genotypic interactions between host and endophyte. Infection frequencies varied among grass populations. We found $62 \%$ of F. rubra populations to have endophyte infections. However, 53 out of 77 populations were either endophyte-free or had very low $(<20 \%)$ infection frequencies. The highest infection frequencies were found in subarctic areas, where infection frequencies were significantly higher in seminatural meadows compared to natural sandy river banks. We found a moderate level of average genotypic diversity and a low level of genetic differentiation $\left(\mathrm{F}_{\mathrm{ST}}=0.0814\right)$ in the 12 populations examined. One dominant genotype occurred in all of the populations, representing $63.5 \%$ of all isolates. Although the existence of a dominant genotype and the detected linkage disequilibrium suggest that the fungus is mainly asexual and vertically transmitted, the multi-allelic loci and variation of genotypic diversity among populations indicate occasional contagious spread and sexual or parasexual recombination of the fungus in some populations. Furthermore, we detected genotypes carrying multi-allelic loci suggesting possibility of multiple infections or hybridisation of the endophyte. An approach to manipulatively generate desired genotype-genotype combinations of the fungus and the plant, and following common garden experiments with naturally endophyte free (E-), naturally endophyte infected $(\mathrm{E}+)$, manipulatively endophyte-free (ME-) and manipulatively endophyte infected $(\mathrm{ME}+)$ plant lineages support the idea of the importance of a genetic mismatch between the fungus and the host as destabilising force in endophyte-grass symbioses. 